\title{
Maxillary Sinus Augmentation With Recombinant Human Bone Morphogenetic Protein-2 and Particulate Allograft Without an Absorbable Sponge: A Case Report
}

Andrew S. Barnett, ${ }^{\star}$ Carlo Maria Soardi, ${ }^{\dagger}$ and Hom-Lay Wang*
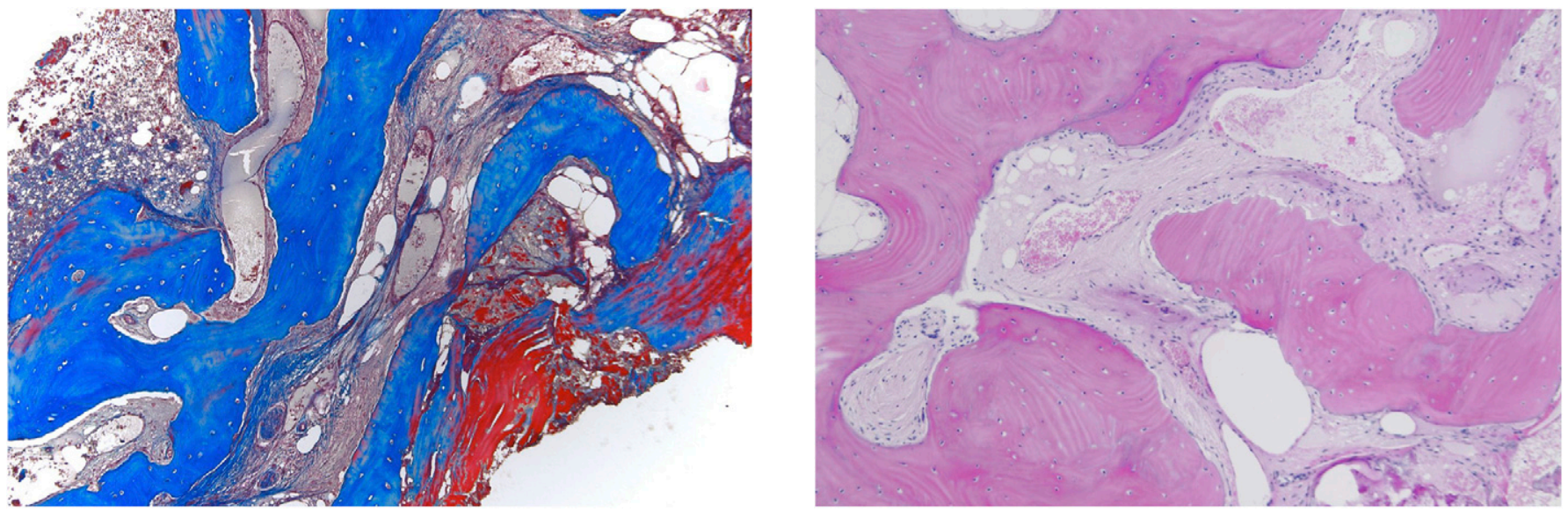

Introduction: Recently, biologic agents have been added to bone graft materials in sinus-augmentation surgeries to accelerate the rate of regeneration. One such agent is recombinant human bone morphogenetic protein-2 (rhBMP-2) using an absorbable collagen sponge (ACS) as a carrier. Researchers have previously shown that, not only can rhBMP-2 be successfully added to an allograft, but that the amount of ACS used is directly related to an increase in future graft shrinkage. There are limited data on adding rhBMP-2 to an allograft mixture without any ACS. Hence, the objective of this case report is to provide the first histomorphometric analysis using rhBMP-2 with allograft mixture without an ACS in an effort to reduce graft shrinkage.

Case Presentation: A patient presented requiring implants in the right posterior, but maxillary sinus pneumatization and ridge resorption resulted in inadequate bone height. A sinus-augmentation procedure was completed using rhBMP-2 with allograft mixture without an ACS in an effort to reduce graft shrinkage; bone core biopsies were trephined at implant placement 6 months later and underwent histologic analysis. The graft regenerated as viable, mature, mineralized bone, and implant placement was successful.

Conclusions: To the best of the authors' knowledge, this is the first report indicating that directly adding rhBMP-2 to an allograft mixture without an ACS carrier is a viable supplementation to a sinus-augmentation procedure. However, longterm randomized controlled trials are needed for proper quantitative analysis and to determine its long-term predictability and efficacy. Clin Adv Periodontics 2014;4:175-181.

Key Words: Allografts; anatomy and histology; bone and bones; collagen; dental implants; dental prosthesis, implantsupported.

\section{Background}

The restoration of the posterior maxilla can often be complicated by the maxillary sinus. Accelerated bone resorption and sinus pneumatization after tooth loss may result in insufficient native bone height to allow for the

\footnotetext{
* Department of Periodontics and Oral Medicine, School of Dentistry, University of Michigan, Ann Arbor, MI.

${ }^{\dagger}$ Private practice, Brescia, Italy.
}

Submitted February 21, 2013; accepted for publication April 4, 2013

doi: 10.1902/cap.2013.130017 proper placement of an implant without compromising its length. Although the current success rates of implants placed in previously augmented maxillary sinuses are already high $\left(90 \%{ }^{1}\right.$ to $\left.91.5 \%^{2}\right)$, the addition of biologic agents with bone graft materials has also been investigated. ${ }^{3-8}$

Bone morphogenetic proteins (BMPs) belong to the transforming growth factor- $\beta$ superfamily and are osteoinductive. ${ }^{9}$ When added to sinus-augmentation procedures, they have been shown to not only improve the quality of the bone graft significantly, but also to accelerate the healing process $^{8,10-12}$ in a dose-dependent manner. ${ }^{13,14}$ Of concern, recombinant human BMP-2 (rhBMP-2) is only approved by the Food and Drug Administration for use with an 


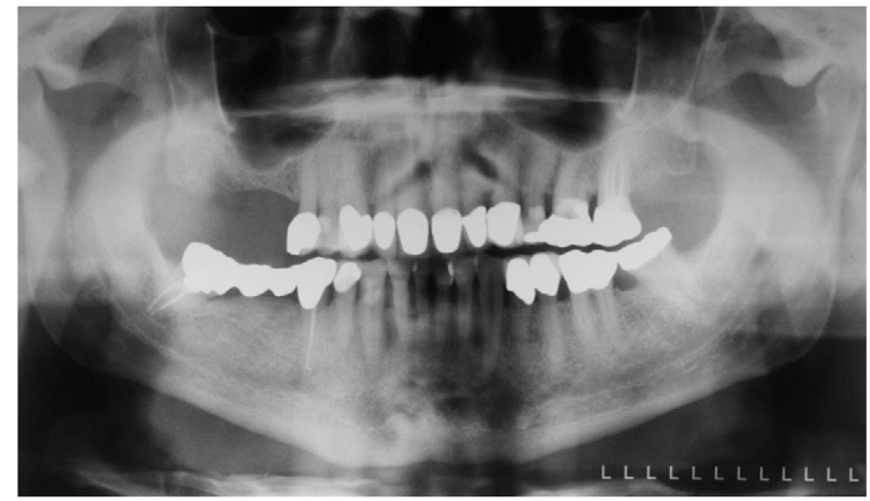

FIGURE 1 Pretreatment panoramic radiograph showing right maxillary sinus pneumatization and ridge resorption.

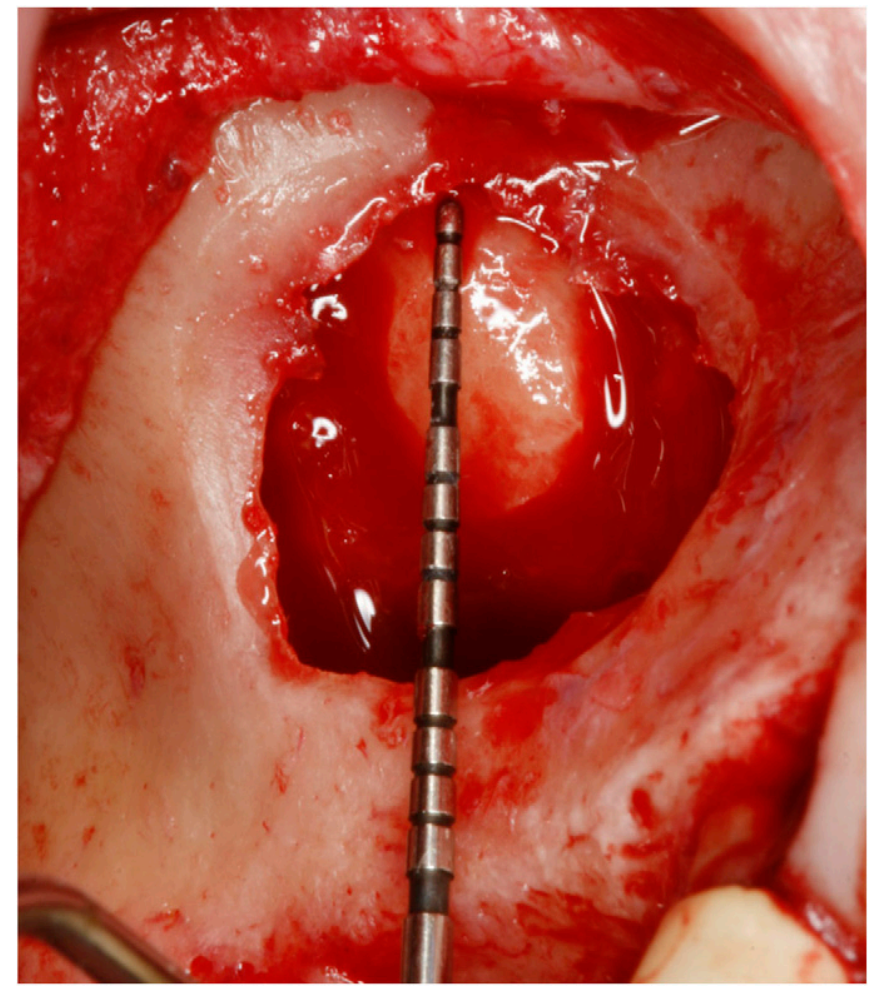

FIGURE 2 Dimensions of the lateral window opening and measurement.

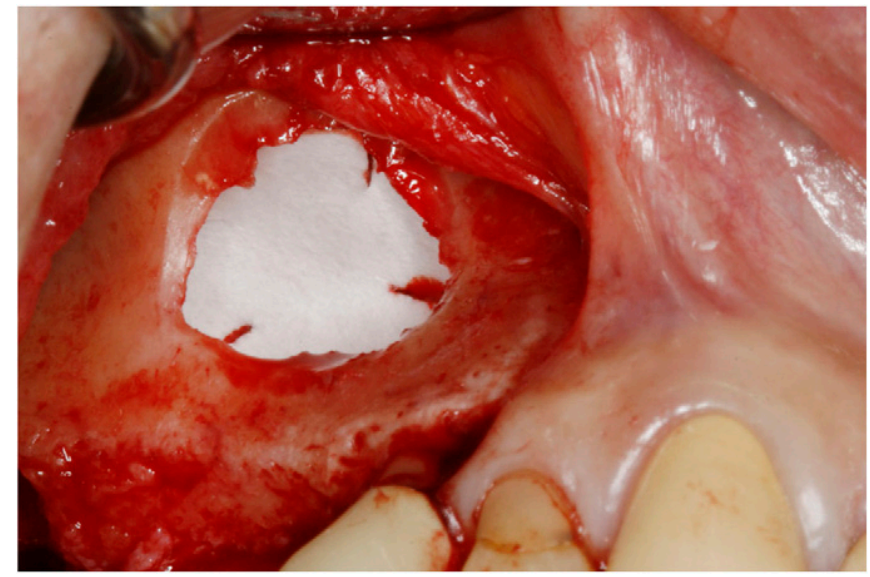

FIGURE 3 Collagen membrane placed against the Schneiderian membrane.

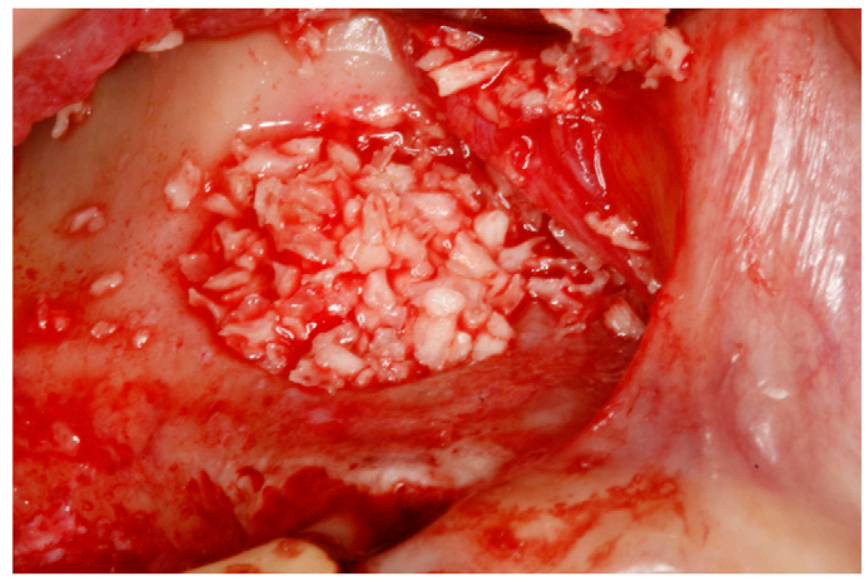

FIGURE 4 Placement of allograft/rhBMP-2 mixture into the sinus cavity.

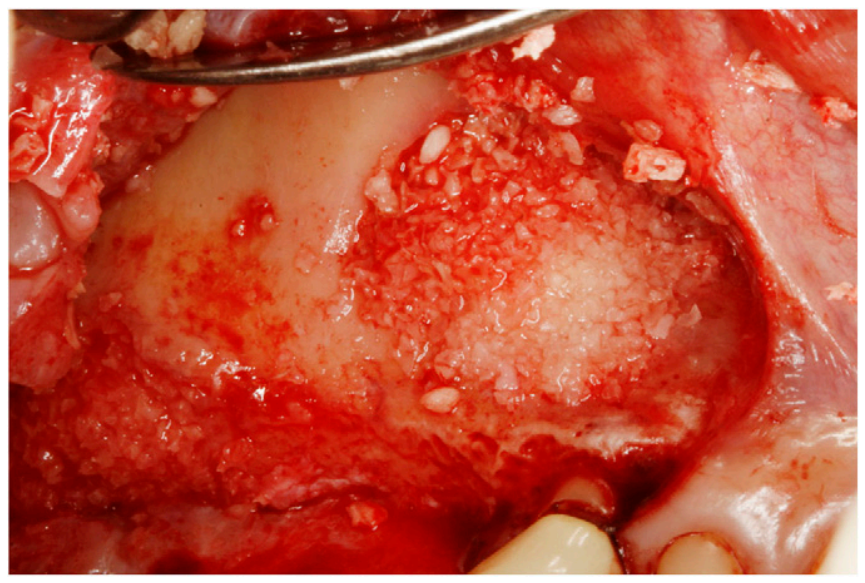

FIGURE 5 Placement of small-particle cancellous allograft to seal the lateral window to promote smooth healing.

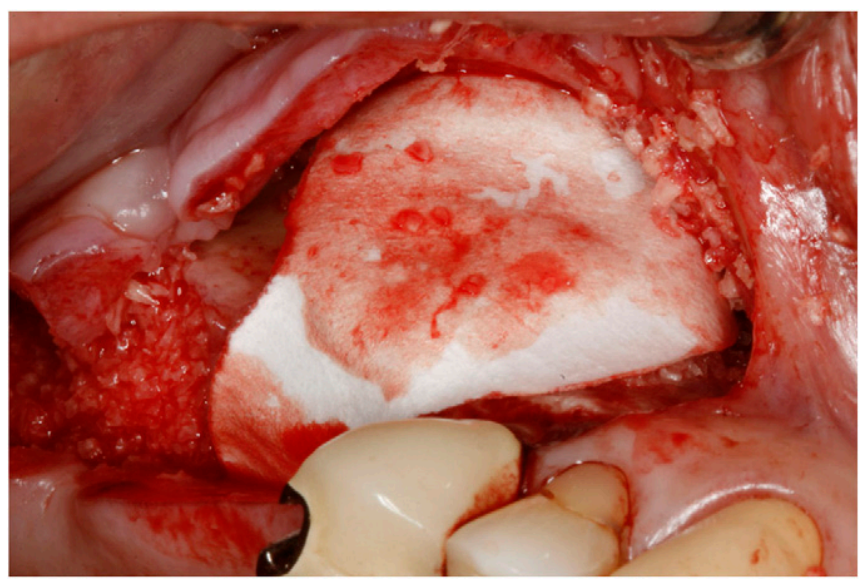

FIGURE 6 Placement of the second absorbable collagen membrane to cover the window and protect the grafts placed.

absorbable collagen sponge (ACS), but researchers have found a direct relationship between the number of sponges used and the degree of shrinkage of the graft material. ${ }^{8}$ If the only approved carrier for rhBMP-2 has poor space maintaining properties in addition to a high resorption rate, then the amount of successful bony augmentation that can be expected is greatly limited. 


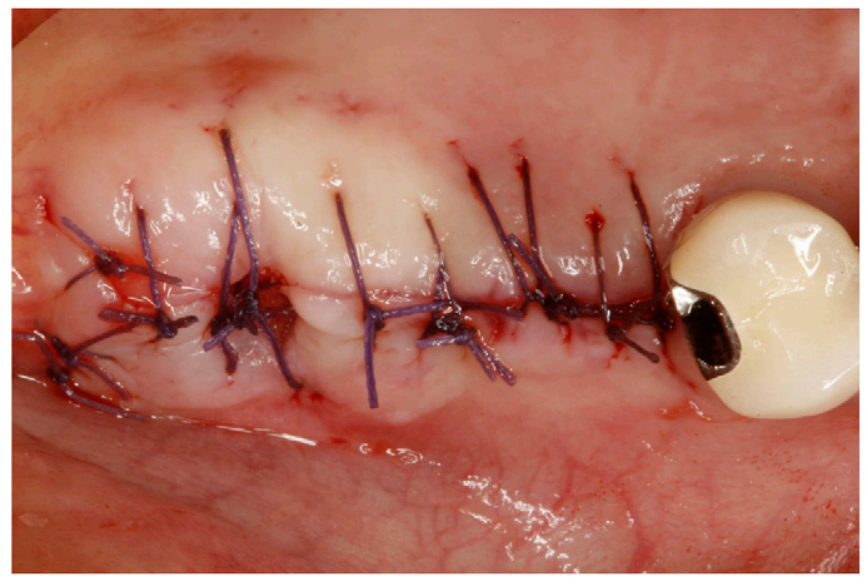

FIGURE 7 Primary flap closure with adequate passivity was achieved with 4-0 polyglactin 910 sutures.

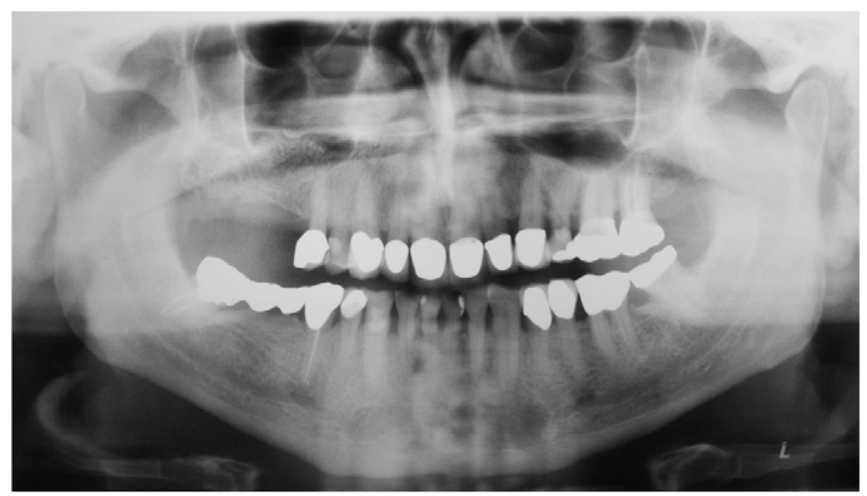

FIGURE 8 Panoramic radiograph at the 2-week postoperative visit.

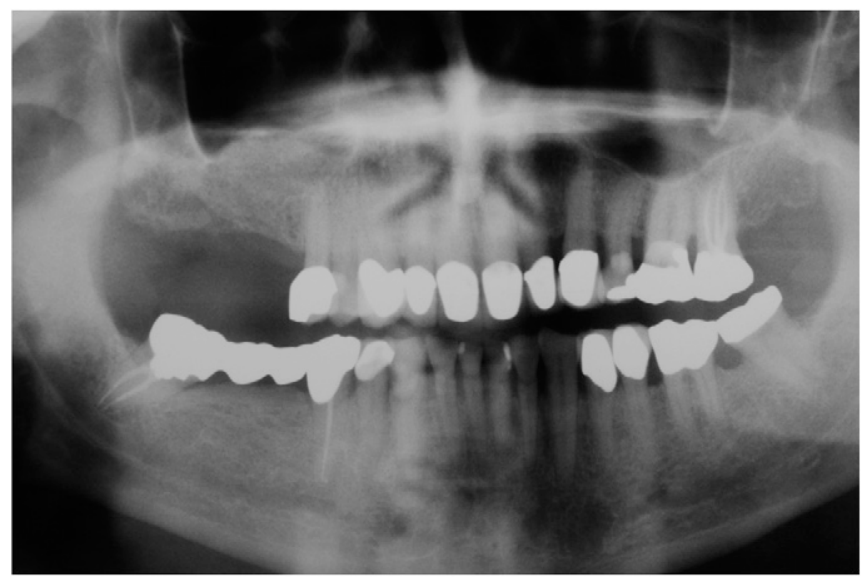

FIGURE 9 Panoramic radiograph at the 6-month postoperative visit demonstrating the graft maturation.

In the current case of a lateral window maxillary sinus augmentation, the authors hoped to optimally reduce the degree of shrinkage by foregoing the use of an ACS and directly adding the rhBMP-2 solution to the allograft mixture.

\section{Clinical Presentation}

On March 2, 2009, a 63-year-old male patient presented to the Dental Faculty Associates clinic at the University of

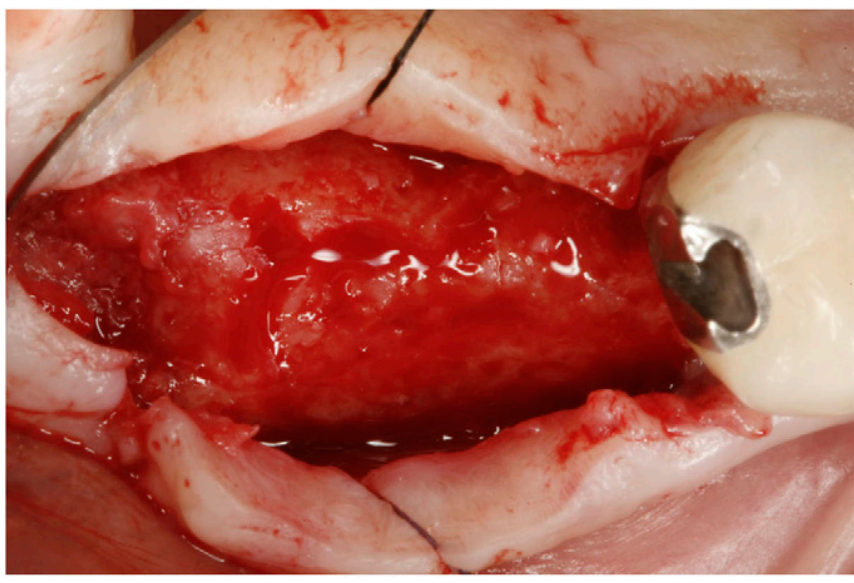

FIGURE 10 Flap reflected, showing good bone formation.


FIGURE 11a Histologic analysis (original magnification $\times 2$ ) using Masson trichrome staining method depicting a greater proportion of mineralized bone (blue) compared with immature osteoid (red). 11b Higher magnification $(\times 20)$ of Masson trichrome staining method showing mineralized bone (blue) compared with immature osteoid (red).

Michigan School of Dentistry, Ann Arbor, Michigan, to restore the edentulous space in his posterior upper right sextant. The patient signed a written informed consent for all treatment. His medical history was significant for osteoarthritis in his hands and a systemic allergy to penicillin. His current medications were daily doses of $20 \mathrm{mg}$ atorvastatin, $325 \mathrm{mg}$ aspirin, and $800 \mathrm{mg}$ ibuprofen. The patient was 

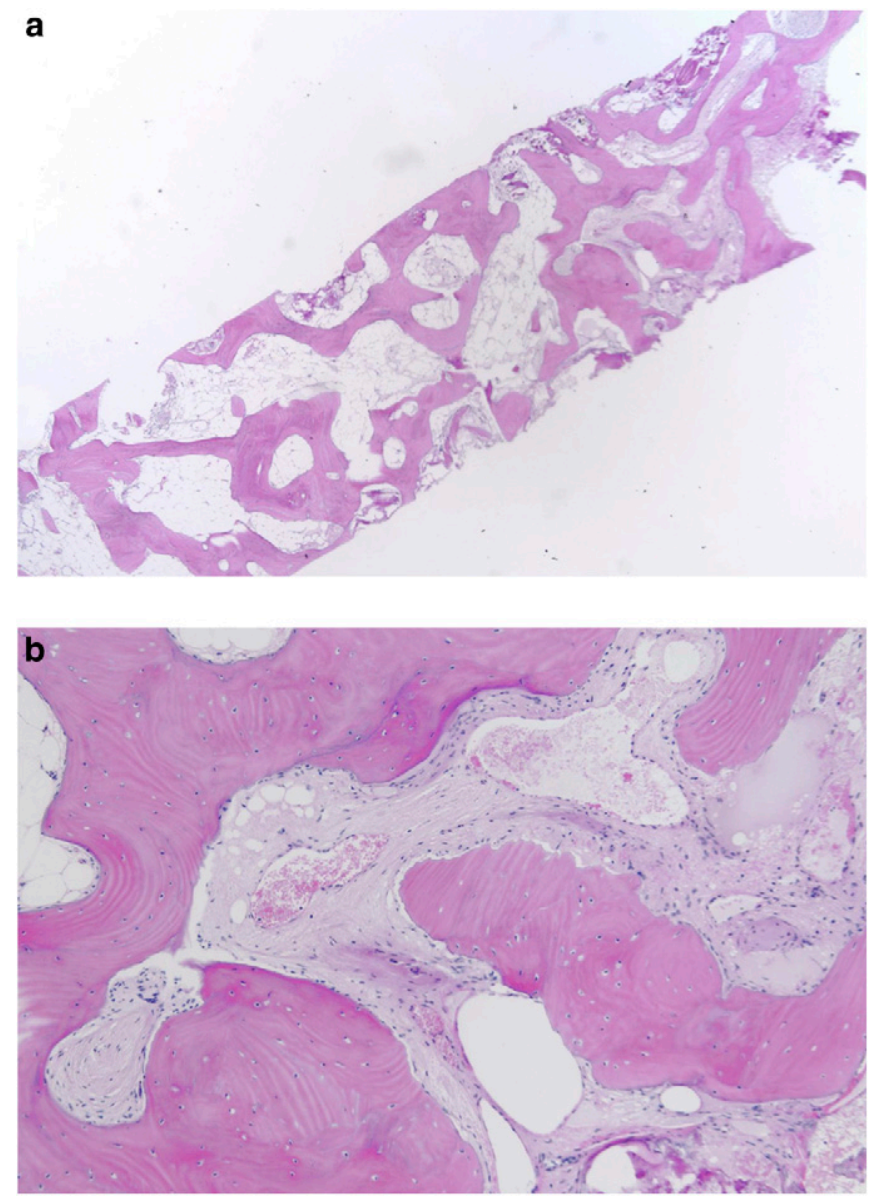

FIGURE 12a Histologic analysis (original magnification $\times 2$ ) using H\&E staining depicting viable osteocytes in newly formed bone. 12b Higher magnification $(\times 20)$ of $\mathrm{H} \& \mathrm{E}$ staining showing viable osteocytes in newly formed bone. classified as American Society of Anesthesiologists Class II physical status. ${ }^{15}$

At the initial appointment, the patient presented with a fixed partial denture for tooth sites \#2 through \#5, with tooth sites \#2, \#4, and \#5 serving as abutments and tooth site \#5 connected through a semirigid connector. Tooth site \#2 had fractured $3 \mathrm{~mm}$ subgingivally and was deemed nonrestorable. The bridge was sectioned distal to tooth site \#4, and tooth site \#2 was extracted with no complications; no ridge preservation was performed at this time. The right sinus demonstrated significant pneumatization and ridge resorption as demonstrated in the pretreatment panoramic radiograph (Fig. 1); in the area of tooth sites \#2 and \#3, mean ridge width was $9.6 \mathrm{~mm}$, and mean ridge height was $3.8 \mathrm{~mm}$.

\section{Case Management}

Seven weeks after extraction, the patient was scheduled for surgery. On the day of surgery, the patient took 500 $\mathrm{mg}$ azithromycin, $800 \mathrm{mg}$ ibuprofen, and $180 \mathrm{mg}$ pseudoephedrine. The patient was placed under moderate intravenous sedation. Local anesthesia was achieved using three carpules of $2 \%$ lidocaine with 1:50,000 epinephrine for local infiltrations. A full-thickness flap was raised and a circular lateral window $10 \mathrm{~mm}$ in diameter was created using high-speed electric and piezoelectric hand pieces $3 \mathrm{~mm}$ apical to the estimated floor of the right maxillary sinus (Fig. 2). The Schneiderian membrane was elevated, and an absorbable collagen membrane ${ }^{\ddagger}$ was placed against it to: 1 ) protect the membrane during bone graft placement; 2) seal any membrane perforations that may

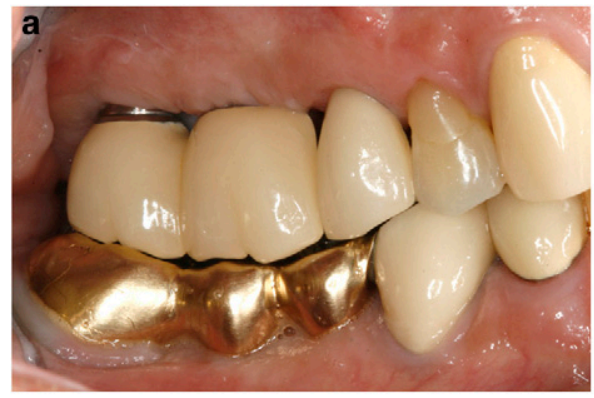

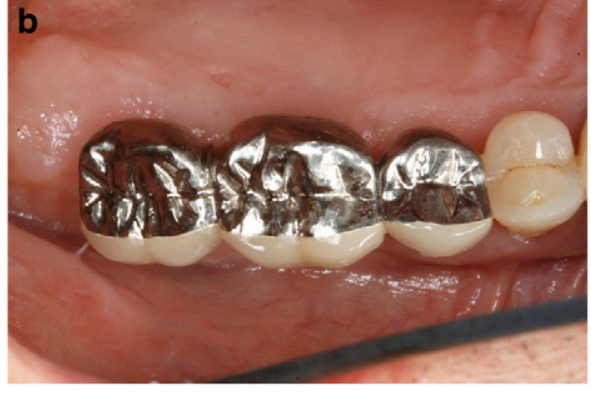



FIGURE 13 Final restoration 6 months after implant placement. 13a Buccal view in occlusion. 13b Occlusal view. 13c Maxillary buccal view. 13d Final periapical radiograph after crown cementation.

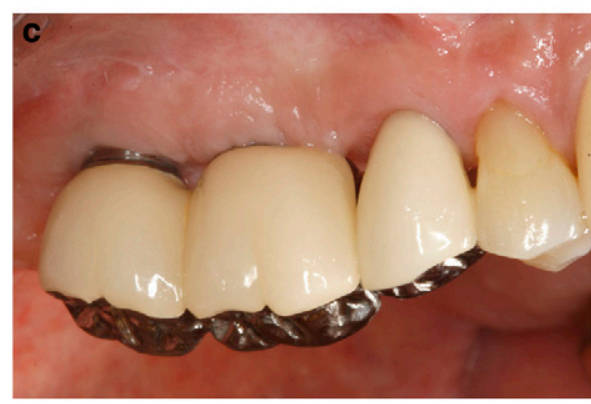

‡ BioMend Collagen Membrane, Zimmer Dental, Carlsbad, CA. 
exist; and 3) allow for better viewing for graft placement (Fig. 3).

A 4:1 mixed ratio of human allograft ${ }^{\S}$ cortical (large particle: 1 to $2 \mathrm{~mm}$ )/cancellous (small particle: 250 to $850 \mu \mathrm{m}$ ) mixture was hydrated with $1.05 \mathrm{mg}$ rhBMP-2" mixed with $1 \mathrm{~mL}$ sterile water. The bone graft mixture was lightly packed into the sinus cavity (Fig. 4). Small-particle mineralized cancellous allograft was used on the most lateral portion of the window to allow for optimal healing (Fig. 5). A second absorbable collagen membrane was trimmed to cover the outside window overlapping the edges by at least 2 to $3 \mathrm{~mm}$ and fixated under the palatal flap (Figs. 6 and 7).

After surgery, medication was prescribed for the patient, including: 1) six tablets of $250 \mathrm{mg}$ azithromycin, two tablets to be taken on the first day and then one tablet for the remaining 4 days; 2) 20 tablets of $800 \mathrm{mg}$ ibuprofen, one tablet to be taken twice daily as needed for pain; 3) nine tablets of $2 \mathrm{mg}$ dexamethasone, four tablets to be taken on the first day, two tablets on the second and third days, and one tablet on the fourth day; and 4) 25 tablets of 10/1,000 mg hydrocodone/acetaminophen, one tablet to be taken three times per day as needed for pain. Healing was uneventful, and sutures were removed after 2 weeks. Panoramic radiographs were taken at the 2-week and 6month postoperative visits (Figs. 8 and 9).

Six months after the sinus augmentation, implant surgery was performed under local anesthesia. A flap was reflected and showed good bone formation (Fig. 10). Trephine bone core biopsies were collected, and two implants ${ }^{\pi}$ were placed at tooth sites \#2 and \#3 $(5.0 \times 13$ and $4.3 \times 13 \mathrm{~mm}$, respectively), achieving excellent primary stability. The patient was placed on a similar regimen of azithromycin and ibuprofen as with the sinus-augmentation surgery.

\section{Clinical Outcomes}

Both core biopsies underwent histologic analyses. Masson trichrome staining method demonstrated the maturation process of the graft (Fig. 11). Immature osteoid is stained red, whereas mature mineralized bone is stained blue. Because there was a significantly greater proportion of mineralized bone (suggesting higher bone density), it can be inferred that the graft underwent regeneration, new bone formation, and maturation. Hematoxylin and eosin (H\&E) staining (Fig. 12) revealed osteocytes within lacunae of the mineralized bone, indicating the viability of the mature bone.

Second-stage surgery was performed after 5 months with sound stability of both implants. Three months later, splinted porcelain fused to metal (PFM) crowns were cemented on implants \#2 and \#3, a PFM crown was cemented on implant \#4, and implant \#5 was restored with a composite restoration. Final restoration was completed 6 months after implant placement and periapical radiographs were taken (Fig. 13).

\section{Discussion}

Comparing panoramic radiographs at 2 weeks and 6 months after the sinus-augmentation procedure revealed minimal density change. Histologic analysis showed significant graft maturation. As such, it appears as though adding rhBMP-2 directly to the allograft mixture is a viable method for maxillary sinus augmentations. However, larger and long-term randomized controlled trials are needed for proper quantitative analysis and to determine its long-term predictability.

\footnotetext{
$\S$ Puros, Zimmer Dental.

"Infuse, Medtronic, Memphis, TN.

ฯ NobelReplace Select implants, Nobel Biocare, Yorba Linda, CA.
} 


\section{Summary}

Why is this case new information? management of this case?
- To the best of the authors' knowledge, this is the first documented case demonstrating the addition of rhBMP-2 directly to an allograft mixture without an ACS, especially with concurrent histologic analysis of the graft.

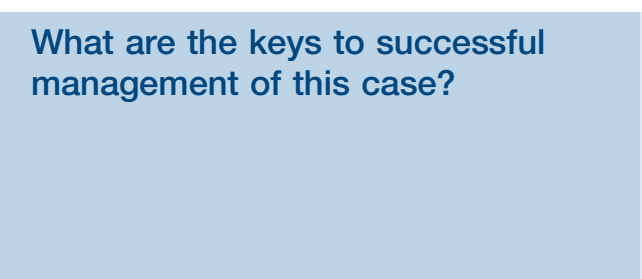

What are the primary limitations to success in this case?
- Patient case selection

- Surgical experience of the clinician

- Avoiding any perforations of the Schneiderian membrane during surgery

- Adequate graft isolation

- Maintaining primary closure postoperatively

- To truly claim the graft regenerated at an accelerated rate with reduced shrinkage attributable to the addition of rhBMP-2 to the allograft, biopsies would need to be taken earlier after the grafting procedure and compared with controls.

\section{Acknowledgments}

This work was partially supported by the University of Michigan Periodontal Graduate Student Research Fund. The authors thank the Oral Pathology Biopsy Service at the University of Michigan School of Dentistry, Ann Arbor, Michigan, for their histologic service and analysis, and Dr. Thomas Marshall, Clinical Assistant Professor, Department of Oral Biologic and Material Sciences/Prosthodontics, University of Michigan School of Dentistry, for his restorative dentistry. Dr. Soardi has received lecture fees from Zimmer Dental, Carlsbad, California. Drs. Barnett and Wang report no conflicts of interest related to this case report.

\section{CORRESPONDENCE:}

Dr. Hom-Lay Wang, Department of Periodontics and Oral Medicine, University of Michigan School of Dentistry, 1011 N. University Ave., Ann Arbor, MI 48109-1078. E-mail: homlay@umich.edu. 


\section{References}

1. Jensen OT, Shulman LB, Block MS, Iacono VJ. Report of the Sinus Consensus Conference of 1996. Int J Oral Maxillofac Implants 1998; 13(Suppl.):11-45.

2. Del Fabbro M, Testori T, Francetti L, Weinstein R. Systematic review of survival rates for implants placed in the grafted maxillary sinus. Int $J$ Periodontics Restorative Dent 2004;24:565-577.

3. Bae JH, Kim YK, Myung SK. Effects of platelet-rich plasma on sinus bone graft: Meta-analysis. J Periodontol 2011;82:660-667.

4. Boyne PJ, Marx RE, Nevins M, et al. A feasibility study evaluating rhBMP-2/absorbable collagen sponge for maxillary sinus floor augmentation. Int J Periodontics Restorative Dent 1997;17:11-25.

5. Froum SJ, Wallace SS, Tarnow DP, Cho SC. Effect of platelet-rich plasma on bone growth and osseointegration in human maxillary sinus grafts: Three bilateral case reports. Int J Periodontics Restorative Dent 2002;22:45-53.

6. Mangano FG, Tettamanti L, Sammons RL, et al. Maxillary sinus augmentation with adult mesenchymal stem cells: A review of the current literature. Oral Surg Oral Med Oral Pathol Oral Radiol 2013; 115:717-723.

7. Nevins ML, Reynolds MA. Tissue engineering with recombinant human platelet-derived growth factor BB for implant site development. Compend Contin Educ Dent 2011; 32:18, 20-17; quiz 28, 40.

8. Tarnow DP, Wallace SS, Testori T, Froum SJ, Motroni A, Prasad HS. Maxillary sinus augmentation using recombinant bone morphogenetic

indicates key references. protein-2/acellular collagen sponge in combination with a mineralized bone replacement graft: A report of three cases. Int J Periodontics Restorative Dent 2010;30:139-149.

9. Urist MR. Bone: Formation by autoinduction. Science 1965;150:893 899.

10. Boyne PJ, Lilly LC, Marx RE, et al. De novo bone induction by recombinant human bone morphogenetic protein-2 (rhBMP-2) in maxillary sinus floor augmentation. J Oral Maxillofac Surg 2005; 63: 1693-1707.

11. Jung RE, Glauser R, Schärer P, Hämmerle CH, Sailer HF, Weber FE. Effect of rhBMP-2 on guided bone regeneration in humans. Clin Oral Implants Res 2003;14:556-568.

12. Jung RE, Thoma DS, Hammerle CH. Assessment of the potential of growth factors for localized alveolar ridge augmentation: A systematic review. J Clin Periodontol 2008;35(Suppl. 8):255-281.

13. Howell TH, Fiorellini J, Jones A, et al. A feasibility study evaluating rhBMP-2/absorbable collagen sponge device for local alveolar ridge preservation or augmentation. Int J Periodontics Restorative Dent 1997;17:124-139.

14. Sigurdsson TJ, Fu E, Tatakis DN, Rohrer MD, Wikesjö UM. Bone morphogenetic protein-2 for peri-implant bone regeneration and osseointegration. Clin Oral Implants Res 1997;8:367-374.

15. American Society of Anesthesiologists. 2013 Relative Value Guide: A Guide for Anesthesia Values. Park Ridge, IL: American Society of Anesthesiologists; 2012 